\title{
Facilitating the Implementation of Distributed Systems with Heterogeneous Interactions
}

\author{
Salwa Kobeissi ${ }^{1}$, Adnan Utayim² ${ }^{2}$, Mohamad Jaber ${ }^{2}$, Yliès Falcone ${ }^{3}$ \\ 1 University of Strasbourg, Inria, ICube Laboratory, Strasbourg, France \\ salwa.kobeissi@inria.fr \\ 2 American University of Beirut, Computer Science Department \\ $\{$ mmu00, mj54\}@aub.edu.lb \\ 3 Univ. Grenoble Alpes, Inria, LIG, F-38000 Grenoble, France \\ ylies.falconeduniv-grenoble-alpes.fr
}

\begin{abstract}
We introduce HDBIP an extension of the Behavior Interaction Priority (BIP) framework. BIP is a component-based framework with a rigorous operational semantics and high-level and expressive interaction model. HDBIP extends BIP interaction model by allowing heterogeneous interactions targeting distributed systems. HDBIP allows both multiparty and direct send/receive interactions that can be directly mapped to an underlying communication library. Then, we present a correct and efficient code generation from HDBIP to C++ implementation using Message Passing Interface (MPI). We present a non-trivial case study showing the effectiveness of HDBIP.
\end{abstract}

\section{Introduction}

Developing correct and reliable distributed systems is challenging mainly because of the complex structures of the interactions between distributed processes. On the one hand, the use of abstract interaction models may simplify the development process but may deteriorate the performance of the generated implementation. On the other hand, the use of low-level primitives makes modeling error prone and time consuming. Although different frameworks $[3,15]$ exist to model interactions between distributed processes, building correct, reliable and scalable distributed systems is still challenging and a hardly predictive task.

In this paper, we introduce HDBIP an extension of the Behavior, Interaction, and Priority (BIP) framework. BIP is a component-based framework used to model heterogeneous and complex systems. BIP has an expressive interaction model [5] that handles synchronization and communication between processes/components. Using only multiparty interactions simplifies the modeling of distributed barriers with local non-determinism, by automatically generating controllers to handle conflicts [6]. Nonetheless, restricting the language to only multiparty interactions affects the performance of the distributed implementations for instance to model a simple asynchronous send/receive primitive. In that case, the implementation requires an explicit buffer component/process. As such, this allows the creation of extra processes that are not needed. This extra buffer is practically duplicated as system buffers are usually provided by the low-level communication libraries (e.g., MPI). Moreover, it is 
required to use multiparty interactions to connect the send primitives and the receive primitives with the explicit buffers. As such, those connections may introduce conflicts between themselves and between multiparty interactions, which may drastically affect the performance of the the distributed implementation.

This paper introduces HDBIP, which allows the modeling of both multiparty and asynchronous send receive interactions in an elegant way. Moreover, we provide an efficient code generation that allows by-construction to directly execute the send receive interactions with no need to create the extra buffers and instead use the system buffers. We show the effectiveness of HDBIP on distributed two-phase commit protocol. We mainly compare with respect to BIP the execution time and the lines of code needed.

The remainder of this paper is structured as follows. Section 2 presents the existing BIP framework. Section 3 introduces HDBIP, an extension of BIP. Section 4 defines how it is possible to generate efficient implementations from HDBIP along with the arguments supporting correctness of the generated implementation. In Section 5, we evaluate the performance of HDBIP by comparing it to BIP. Section 6 presents related work. Finally, Section 7 draws some conclusions and presents future work.

\section{Behavior Interaction Protocol (BIP) Framework}

The Behavior Interaction Priority (BIP) framework [3] offers high-level synchronization primitives that simplify system development and allow for the generation of both centralized and distributed implementations from high-level models. It consists of three layers: Behavior, Interaction and Priority. Behavior is expressed by Labeled Transition Systems (LTS) describing atomic components extended with data and $\mathrm{C}$ functions. Moreover, transitions of atomic components are labeled with ports that are exported for communication/synchronization with other components. Interaction models the synchronization and communication between ports of atomic components. Priority specifies scheduling constraints on interactions.

\subsection{Atomic Components}

Let us consider a set of local variables $X$.

Definition 1 (Port). A port is a tuple $\left\langle p, X_{p}\right\rangle$ where $p$ is an identifier and $X_{p} \subseteq X$ is a set of exported local variables. A port is referred to by its identifier.

Definition 2 (Atomic component - Syntax). An atomic component is a tuple $\left\langle P, L, T, X,\left\{g_{\tau}\right\}_{\tau \in T},\left\{f_{\tau}\right\}_{\tau \in T}\right\rangle$, such that:

- $\langle P, L, T\rangle$ is an LTS over a set of ports $P, L$ is a set of control locations, and $T \subseteq L \times P \times L$ is a set of transitions;

- $X$ is a finite set of variables;

- Every transition $\tau \in T$ has a guard $g_{\tau}$ (a predicate over $X$ ), and a function $f_{\tau} \in$ $\left\{x:=f^{x}(X) \mid x \in X\right\}^{*}$, triggered by this transition, that updates the values of variables in $X$. 


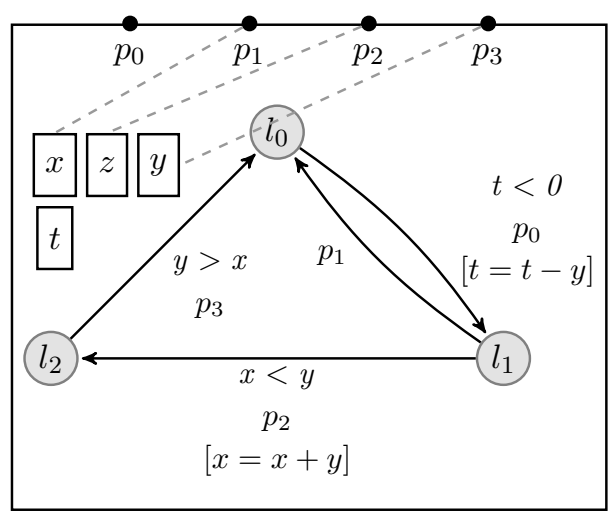

Fig. 1: Atomic component in BIP

A transition $\tau=\left\langle l, p, l^{\prime}\right\rangle \in T$, where $l$ (resp. $l^{\prime}$ ) is the source (resp. destination) of $\tau . p$ is the label of $\tau$ used as an interface to synchronize with other components. Moreover, a transition can be augmented with a guard $g_{\tau}$ and a function $f_{\tau}$, thus defined as $\tau=\left\langle l, p, g_{\tau}, f_{\tau}, l^{\prime}\right\rangle$. The port attached to a transition is said to be enabled only if the guard of the transition $g_{\tau}$ holds.

Definition 3 (Atomic component - semantics). The semantics of atomic component $\left\langle P, L, T, X,\left\{g_{\tau}\right\}_{\tau \in T},\left\{f_{\tau}\right\}_{\tau \in T}\right\rangle$ is the LTS $\left\langle Q, P, T_{0}\right\rangle$, where:

- $Q=L \times[X \rightarrow$ Data $] \times(P \cup\{$ null $\})$;

- $T_{0}=\left\{\left\langle\langle l, v, p\rangle, p^{\prime}\left(v_{p^{\prime}}\right),\left\langle l^{\prime}, v^{\prime}, p^{\prime}\right\rangle\right\rangle \in Q \times P \times Q \mid \exists \tau=\left\langle l, p^{\prime}, l^{\prime}\right\rangle \in T\right.$ : $\left.g_{\tau}(v) \wedge v^{\prime}=f_{\tau}\left(v / v_{p^{\prime}}\right)\right\}$, where $v_{p^{\prime}} \in\left[X_{p^{\prime}} \rightarrow\right.$ Data $]$.

A configuration/state of an atomic component is a triple $\langle l, v, p\rangle \in Q$ where $l \in L$, $v \in[X \rightarrow$ Data $]$ is a valuation of variables in $X$, and $p \in P$ is the port of the lastexecuted transition (or null otherwise, i.e., in case of the initial configuration). The evolution $\langle l, v, p\rangle \stackrel{p^{\prime}\left(v_{p^{\prime}}\right)}{\rightarrow}\left\langle l^{\prime}, v^{\prime}, p^{\prime}\right\rangle$, where $v_{p^{\prime}}$ is a valuation of the variables in $X_{p^{\prime}}$, is possible if there exists a transition $\left\langle l, p^{\prime}, g_{\tau}, f_{\tau}, l^{\prime}\right\rangle$, s.t. $p^{\prime}$ is enabled or $g_{\tau}(v)=$ true. Valuation $v$ is modified to $v^{\prime}=f_{\tau}\left(v / v_{p^{\prime}}\right)$.

We use the dot notation to denote the elements of an atomic component $B$. For instance, we refer to its set of ports as B.P, its set of locations as B.L and its set of local variables as $B . X$.

Figure 1 depicts an atomic component $B . B$ has four ports $p_{0}, p_{1}, p_{2}$ and $p_{3}$ and four local variables $x, y, z$ and $t$. Port $p_{1}$ exports variable $x, p_{2}$ exports $z$, and $p_{3}$ exports $y$. In addition, $B$ has three locations $\ell_{0}, \ell_{1}$ and $\ell_{2}$ with initial location $\ell_{0}$. Each transition between locations has a guard, a port and an update function or the computation to be applied. For example, the transition between locations $\ell_{1}$ and $\ell_{2}$ is labeled by port $p_{2}$ and guarded by $x<y$ and applies computation $(x=x+y)$ when executed. When this transition is executed, the value of $z$ exported by $p_{2}$ is changed according to the valuation received through $p_{2}$. 


\subsection{Composite Components}

We consider a set of atomic components $\left\{B_{i}\right\}_{i \in I}$ with $I \subseteq[1, n]$ and $B_{i}=$ $\left\langle P_{i}, L_{i}, T_{i}, X_{i},\left\{g_{\tau}\right\}_{\tau \in T_{i}},\left\{f_{\tau}\right\}_{\tau \in T_{i}}\right\rangle$, where atomic components have disjoint sets of locations, variables and ports, i.e., for all $i, j \in I$ such that $i \neq j, L_{i} \cap L_{j}=\emptyset$, $P_{i} \cap P_{j}=\emptyset$ and $X_{i} \cap X_{j}=\emptyset$. We denote the set of all ports (resp. locations, variables) of a composite component by $P=\bigcup_{i \in I} P_{i}$ (resp. $L=\bigcup_{i \in I} L_{i}, X=\bigcup_{i \in I} X_{i}$ ). Atomic components synchronize and exchange data through interactions.

Definition 4 (Interaction). An interaction is defined as a tuple $a=\left\langle P_{a}, G_{a}, F_{a}\right\rangle$, where:

- $P_{a}$ is a non-empty set such that $P_{a} \subseteq P$, and, for every $i \in I\left|P_{i} \cap P_{a}\right| \leq 1$, i.e., an interaction a consists of at most one port of every atomic component in $B$;

- $G_{a}$ is a guard over valuation of $X_{a}$, where $X_{a}$ are the variables attached to ports $P_{a}$; and

- $F_{a}$ is an update function over the valuation of $X_{a}$.

We denote the ports associated in an interaction $a$ as $P_{a}=\left\{p_{i}\right\}_{i \in I}$ where $i$ is the identification index of the atomic component because at most one port of every atomic component can be included in the same interaction. Moreover, an interaction can include variables that are denoted as $X_{a}=\bigcup_{p \in P_{a}} X_{p}$. The updated value of $X_{p_{i}}$, transferred to $B_{i}$ as an interaction outcome, after projecting the update function $F_{a}$ is denoted as $F_{a_{i}}$.

Definition 5 (Composite component). A composite component $C$ consists in applying a set of interactions $\gamma$ to a set of distinct atomic components $\left\{B_{i}\right\}_{i \in I}$ with $I \subseteq[1, n]$. Therefore, a composite component $C$ is defined as $\gamma\left(\left\{B_{i}\right\}_{i \in I}\right)$

Figure 2 shows an example of a composite component $C=\gamma\left(\left\{B_{1}, B_{2}, B_{3}\right\}\right)$ where $B_{1}, B_{2}$ and $B_{3}$ are atomic components, and $\gamma=\left\{a_{1}, a_{2}, a_{3}, a_{4}, a_{5}\right\}$.

Definition 6 (Semantics of composite components). A state $q$ of composite component $C=\gamma\left(\left\{B_{1}, \ldots, B_{n}\right\}\right)$ is an n-tuple $\left\langle q_{1}, \ldots, q_{n}\right\rangle$ where $q_{i}=\left\langle l_{i}, v_{i}, p_{i}\right\rangle$ is a state of $B_{i}$. The semantics of $C$ is an LTS $S_{c}=\langle Q, \gamma, \longrightarrow\rangle$, where:

- $Q=B_{1} \cdot Q \times \ldots \times B_{n} \cdot Q$

- $\gamma$ is the set of all possible interactions; and

$-\longrightarrow$ is the least set of transitions satisfying the following rule: $\exists a \in \gamma: a=\left\langle\left\{p_{i}\right\}_{i \in I}, G_{a}, F_{a}\right\rangle \quad G_{a}\left(v\left(X_{a}\right)\right)$

$$
\frac{\forall i \in I: q_{i}{\stackrel{p_{i}\left(v_{i}\right)}{\longrightarrow}}_{i} q_{i}^{\prime} \wedge v_{i}=F_{a_{i}}\left(v\left(X_{a}\right)\right) \quad \forall i \notin I: q_{i}=q_{i}^{\prime}}{\left\langle q_{1}, \ldots, q_{n}\right\rangle \stackrel{a}{\longrightarrow}\left\langle q_{1}^{\prime}, \ldots, q_{n}^{\prime}\right\rangle}
$$

$X_{a}$ is the set of variables attached to the ports of $a, v$ is the global valuation. $F_{a_{i}}$ is the projection of $F$ to the variables of $p_{i}$ yielding to the valuation $v_{p_{i}}$ of the variables in $X_{i}$ exported by $p_{i}$.

The above rule means that whenever all the ports of an interaction $a$ are enabled and the guard corresponding to $a,\left(G_{a}\left(v\left(X_{a}\right)\right)\right)$ holds, $a$ is enabled. One enabled interaction 


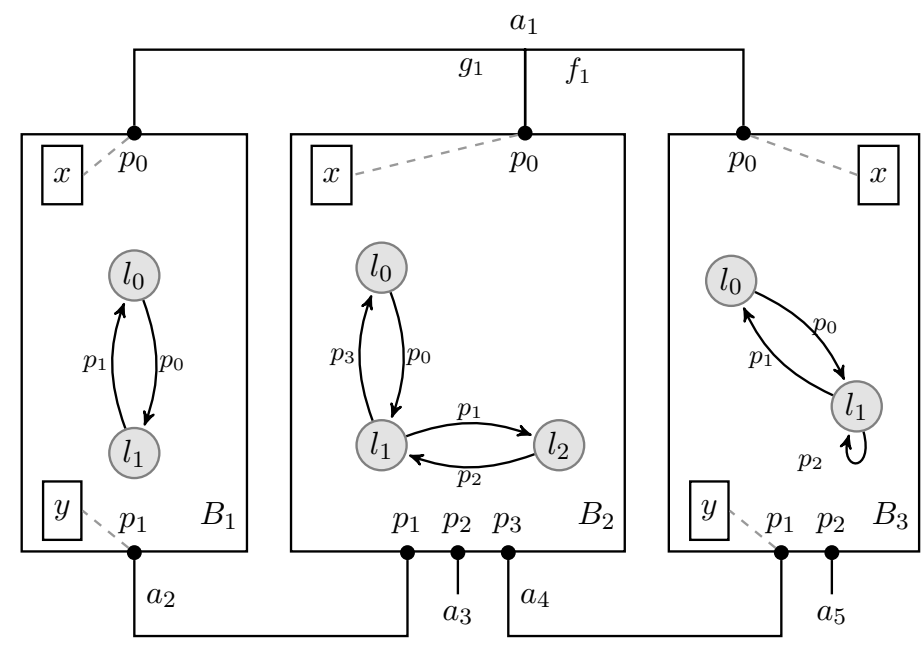

Fig. 2: Composite component in BIP

is selected and the state of the components whose ports are involved in the interaction $a$ changes by executing location function and moving to the next set of locations. The state of the components that are not involved in this interaction remain unchanged. A straightforward implementation of this semantics can be realized by a centralized engine that allows the execution of one enabled interaction at a time. Note, practically, it is also possible to concurrently execute independent interactions (which do not share components), while preserving the above semantics.

Figure 2 represents a composite component $\mathcal{C}$ made up of three components atomic $=\left\{B_{1}, B_{2}, B_{3}\right\}$ by applying a set of five interactions $\gamma=\left\{a_{1}, a_{2}, a_{3}, a_{4}, a_{5}\right\}$. For instance, interaction $a_{1}$ is enabled when all of its involved ports, i.e., $B_{1} \cdot p_{0}, B_{2} . p_{0}$ and B3. $p_{0}$, are enabled and its corresponding guard $g_{1}$ holds. But, in this example, the ports are not associated with guards which means that by default all ports are enabled. Assuming that guard of $a_{1}$ holds, this interaction is said to be enabled. In case it is selected to execute, its function $f_{1}$ is also applied upon its execution. Furthermore, upon the execution of $a_{1}$, transitions $\left\langle B_{1} . l_{0}, B_{1} \cdot p_{0}, B_{1} . l_{1}\right\rangle,\left\langle B_{2} \cdot l_{0}, B_{2} \cdot p_{0}, B_{2} \cdot l_{1}\right\rangle$, $\left\langle B_{3} . l_{0}, B_{3} . p_{0}, B_{3} \cdot l_{1}\right\rangle$ will, also, execute for their ports are involved in $a_{1}$.

\subsection{Distributed Implementation - Send/Receive BIP}

A high-level BIP model can be transformed into a distributed implementation to achieve parallelism between components and interactions [6]. To do so, a BIP model is transformed into its equivalent send/receive BIP. Send/receive BIP consists of three layers: (1) an atomic components layer that consists of atomic components transformed to interact with the upper layer to execute multiparty interactions; (2) an interaction layer that consists of components responsible to execute interactions; (3) a conflict resolution layer that is responsible to forbid the concurrent execution of two conflicting interactions (to preserve the semantics of the initial model). The 
obtained model consists of transforming multiparty interactions into send/receive communication protocols. More precisely, each transition of atomic components is split in two transitions: (1) send offering, which sends the enabled ports to the components (that are handling the interactions corresponding to enabled ports) in the interaction layer; (2) receive, which waits for an acknowledgment from the interaction layer to execute the selected port. As such, the interaction protocol collects all enabled ports and determines what are the enabled interactions. As the interaction layer consists of several components handling different interactions, it is possible that two conflicting interactions are marked to be enabled by different components of the interaction layer, which may lead to the concurrent execution of two conflicting interactions. To remedy this, the interaction layer consults first with the conflict resolution, which is responsible for handling conflicts between interactions. Note, two interactions are said to be conflicting iff either: (1) there is a common port involved in them, or (2) if they include two distinct ports belonging to the same component where those ports are the label of two distinct transitions outgoing from the same source location.

Remark. Implementing a system with multiparty interactions requires solving potential conflicts, which is addressed in [6] for systems without priorities and in [7] for systems with priorities. Independently, we focus on those interactions that can be realized by asynchronous send/receive communication over multiparty interaction. For the sake of simplicity, and without lost of generality we consider systems without priorities.

\section{Heterogeneous Distributed BIP - HDBIP}

BIP uses multiparty interactions to model communication and synchronization between components, which is expressive enough to model any communication or synchronization primitives [5]. Nonetheless, modeling a simple asynchronous send/receive primitive requires to (1) explicitly create components representing buffers; (2) create intermediate schedulers to coordinate the execution of the interactions. This may drastically affect the performance of the generated distributed implementations. To overcome this, we introduce HDBIP that combines both multiparty and direct asynchronous send/receive (DASR) interactions. This simplifies the modeling of distributed systems and allows for efficient code generation. For instance, implementing DASR primitives can benefit from the underlying primitives such as system buffers and does not require to create extra components for scheduling with other interactions (i.e., conflict-resolution) or for buffer modeling. The components composing the HDBIP model are known as partially asynchronous (PA) atomic components.

\subsection{HDBIP Syntax}

A PA atomic component $B^{\star}$ is a regular BIP atomic component where transitions are labeled with three types of ports: ordinary, direct send and direct receive: (1) ordinary ports are the same to those defined in BIP; (2) direct send ports are used to model asynchronous direct communication with receive ports. Hereafter, we represent ordinary, direct send and direct receive ports, by black circle, blue rectangle and red diamond, respectively. 


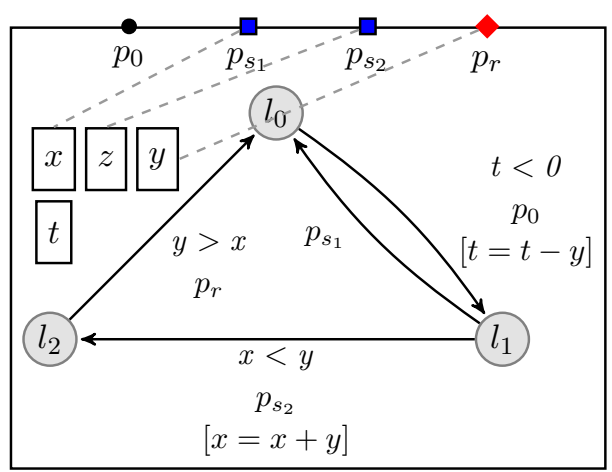

Fig. 3: Atomic component in HDBIP

Definition 7 (Partially Asynchronous Atomic component). A PA atomic component $B^{\star}$ is tuple $\langle B, t\rangle$ where:

- $B$ is an atomic component;

- $t: P \rightarrow\{$ ordinary, send, receive $\}$ is a function that maps ports to their types.

Figure 3 depicts a PA atomic component $B^{\star}$ in HDBIP. $B^{\star}$ has four ports $p_{0}, p_{s_{1}}$, $p_{s_{2}}$, and $p_{r}$ and four local variables $x, y, z$, and $t$. Port $p_{s_{1}}$ exports $x, p_{s_{2}}$ exports $z$ and $p_{r}$ exports $y$. In addition, $B$ has three locations $\ell_{0}, \ell_{1}$ and $\ell_{2}$ with initial location $\ell_{0}$. Hereafter, we consider a set of PA atomic components $\left\{B_{i}^{\star}\right\}_{i \in I}$, where $\forall i \in I, B_{i}^{\star}=$ $\left\langle B_{i}, t_{i}\right\rangle$. Let $P_{o}=\bigcup_{i \in I} B_{i}^{\star} \cdot P_{o}$ (resp. $P_{s}, P_{r}, P$ ) denotes the set of all the ordinary (resp. direct send, direct receive, all) ports. Moreover, without loss of generality, we assume that from any location, the outgoing transitions can be labeled with both ordinary and send or receive ports (i.e., either only ordinary ports or a mix of send or receive ports). This allows to efficiently generate distributed implementation and makes the interaction model not ambiguous to the developers of the atomic components. Note that the transitions requirements hold in the PA component depicted in Figure 3.

We distinguish two types of interactions: (1) ordinary; and (2) DASR. Ordinary interaction is the same as regular BIP interaction, i.e., allows to model multiparty interaction. Hence, it connects ordinary ports. DASR interaction allows to model asynchronous send receive interaction and connects a sender port of a component to receiver ports of different components.

Definition 8 (Ordinary Interaction). An ordinary interaction a is defined by the tuple $\left\langle P_{a}, G_{a}, F_{a}\right\rangle$ where:

- $P_{a} \subseteq P$ is a non-empty set such that $P_{a} \subseteq P_{o}$ and, $\forall i \in I,\left|B_{i}^{\star} . P \cap P_{a}\right| \leq 1$; and

- $G_{a}$ and $F_{a}$ are the guard and the function of the ordinary interaction, the same as the ones defined in the BIP interaction.

Definition 9 (DASR Interaction). A DASR interaction a is defined by $P_{a}$ where:

- $P_{a} \subseteq P$, with $\left|P_{a}\right|>1$, is a set such that $\left|P_{a} \cap P_{s}\right|=1,\left|P_{a} \cap P_{o}\right|=0,\left|P_{a} \cap P_{r}\right|>0$ and, $\forall i \in I,\left|P_{i} \cap P_{a}\right| \leq 1$; 


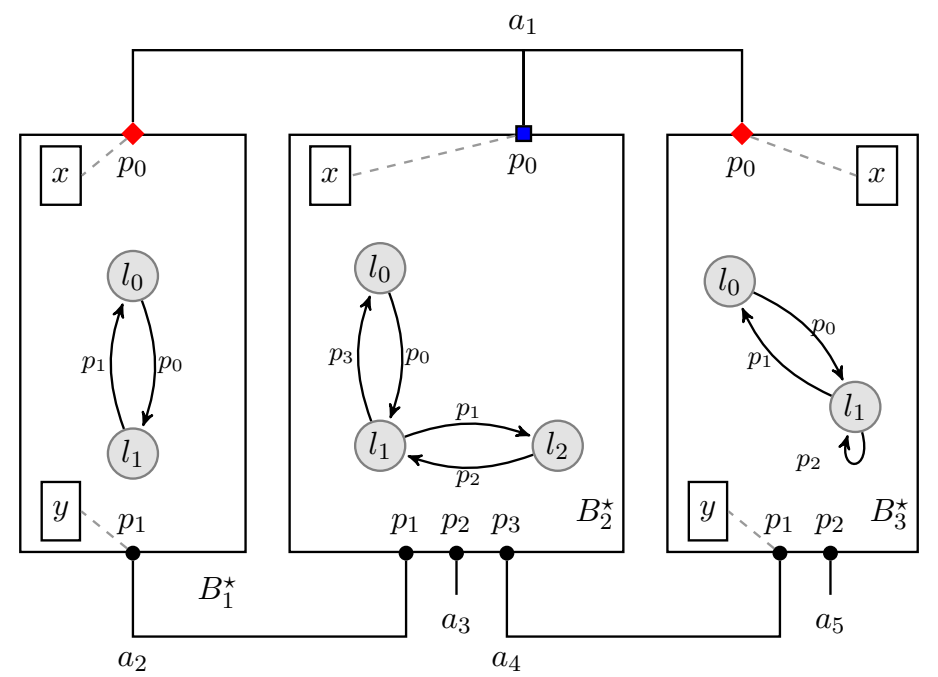

Fig. 4: Composite component in HDBIP

- all ports have the same type; (3) its guard is always hold, however, its send port can have a local guard;

- its function allows only for data transfer of data attached to the sender port to the data attached to the receiver ports.

Note that a send port can only participate in one DASR interaction, whereas a receive port can participate in several DASR interactions.

Definition 10 (Partially asynchronous composite component). A PA composite component $C^{\star}$ denoted by $\gamma^{\star}\left(\left\{B_{i}^{\star}\right\}_{i \in I}\right)$ consists of a set of atomic components $\left\{B_{i}^{\star}\right\}_{i \in I}$ composed by applying a set of ordinary and DASR interactions $\gamma^{\star}$

Given a PA composite component $\gamma^{\star}\left(\left\{B_{i}^{\star}\right\}_{i \in I}\right)$ where $B_{i}^{\star}=\left\langle B_{i}, t_{i}\right\rangle$ for all $i \in I$, we define:

- type : $\gamma^{\star} \rightarrow\{$ ordinary, sendreceive $\}$ is a function that maps interactions to their types;

- $\gamma_{o}=\left\{a \in \gamma^{\star} \mid \operatorname{type}(a)=\right.$ ordinary $\}$ the set of all ordinary interactions; and

- $\gamma_{s r}=\left\{a \in \gamma^{\star} \mid \operatorname{type}(a)=\right.$ sendreceive $\}$ the set of all DASR interactions.

Clearly, $\gamma^{\star}=\gamma_{o} \cup \gamma_{s r}$ and $\gamma_{o} \cap \gamma_{s r}=\emptyset$. Figure 4 depicts a PA composite component made up of a set of three PA components $B^{\star}=\left\{B_{1}^{\star}, B_{2}^{\star}, B_{3}^{\star}\right\}$ by applying a set of five interactions $\gamma^{\star}=\left\{a_{1}, a_{2}, a_{3}, a_{4}, a_{5}\right\}$, where only $a_{1}$ is a DASR interaction while the rest $\left(a_{2}, a_{3}, a_{4}\right.$ and $\left.a_{5}\right)$ are ordinary interactions. $a_{1}$ is a DASR interaction because it consists of a direct send port $B_{2}^{\star} \cdot p_{0}$ and the receive ports $B_{1}^{\star} \cdot p_{0}$ and $B_{3}^{\star} \cdot p_{0} . a_{1}$ is said to be a valid DASR interaction because it does not include any ordinary port. Moreover, $B_{2}^{\star} \cdot p_{0}$ cannot participate in further interactions. 


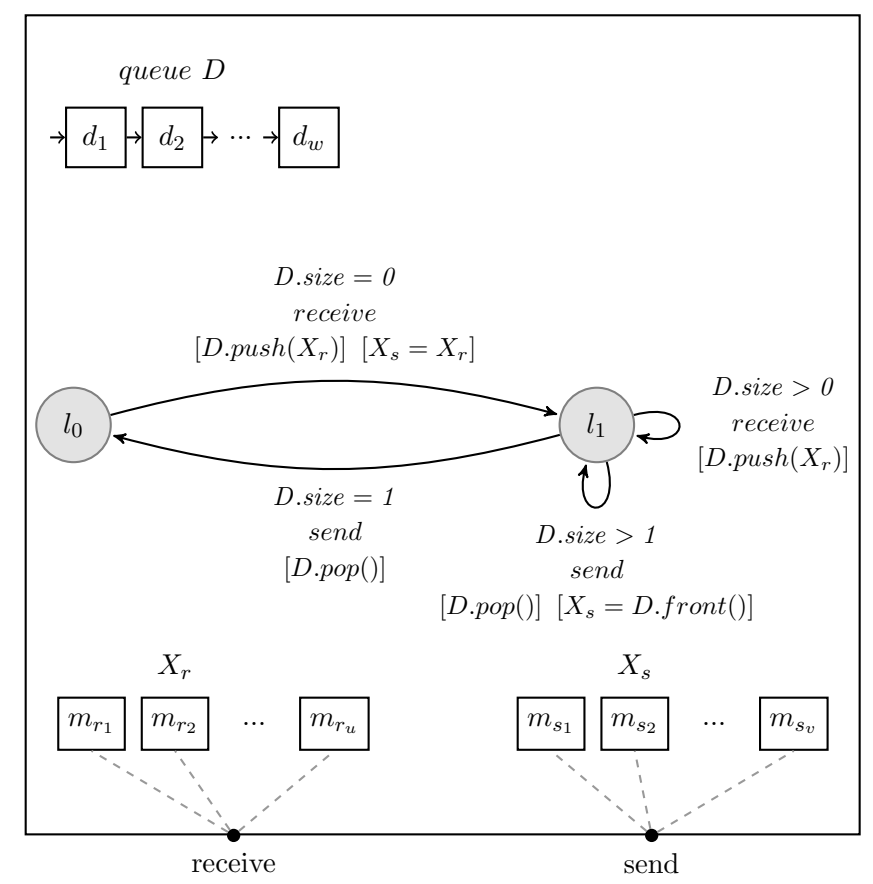

Fig. 5: Buffer component

\subsection{HDBIP Semantics}

We define the semantics of a PA composition component $C^{\star}$ by transforming it into its equivalent BIP model $C=\llbracket C^{\star} \rrbracket$. The transformation consists of the following steps: (1) create buffer atomic components; (2) create interactions connecting send and receive ports with buffer components.

Creating Buffer components. We first create a buffer component $B u_{p}^{i}$ for every receive port $p \in \bigcup_{i \in I} B_{i}^{\star}$.Pr. $B u^{i}$ is an atomic component where: (1) $B u . X=$ $X_{s} \cup X_{r} \cup D$ such that $D$ is a queue that can hold data of the same type of the port, $X_{s}=\left\{x_{s} \mid x \in p . X\right\}$ is the set of received variables that correspond to port $p$, and $X_{r}=\left\{x_{r} \mid x \in p . X\right\}$ is the set of send variables that correspond to port $p$; (2) Bu.P $=\{$ send,receive $\}$, where send port exports the set of variables $X_{s}$, and receive port exports the set $X_{r}$; (3) Bu.L $=\left\{l_{0}, l_{1}\right\}$, where $l_{0}$ is the initial location; (4) Bu.T $=\left\{\tau_{1}, \tau_{2}, \tau_{3}, \tau_{4}\right\}$ such that $\tau_{1}=\left\langle l_{0}\right.$, receive, $\left.l_{1}\right\rangle, \tau_{2}=\left\langle l_{1}\right.$, receive, $\left.l_{1}\right\rangle$, $\tau_{3}=\left\langle l_{1}\right.$, send,$\left.l_{1}\right\rangle$ and $\tau_{4}=\left\langle l_{1}\right.$, send, $\left.l_{0}\right\rangle$. The guards of transitions are predicates over the queue $D$ and its size. Assuming the queue size can be denoted as D.size, guard $g_{1}$ of $\tau_{1}$ is D.size $=0, g_{2}$ of $\tau_{2}$ is D.size $>0, g_{3}$ of $\tau_{3}$ is D.size $>1$, and, finally, $g_{4}$ of $\tau_{4}$ is D.size $=1$. Yet, the size of the queue is not determined by the size of the message received, but by the number of messages received. The functions, on transitions including the port receive, from $l_{0}$ to $l_{1}$, involve adding the values of $X_{r}$ (as one list) 
to the list $D$ and updating the values of $X_{s}$ to that of $X_{r}$, whereas, from $l_{1}$ to $l_{1}$, the values of $X_{r}$ are only added to $D$. Initially, in $\tau_{1}, X_{s}$ is updated to the values of the first received message. Thus, the functions, on transitions including the port send, from $l_{1}$ to $l_{1}$, involve removing data from the list $D$ first, then updating the values of $X_{s}$ to be the oldest list of values received and pushed to $D$. On the other hand, from $l_{1}$ to $l_{0}$, only the last list of values in $D$ is removed emptying $D$. The set of all buffers for all receive ports in $C^{\star}$ is denoted by $B U=\bigcup_{i \in I}\left\{B u_{p}^{i} \mid p \in B_{i}^{\star} . P \wedge t_{i}(p)=\right.$ receive $\}$. Note that port receive of the buffer is always enabled, i.e., its guard is true, whereas port send is enabled when there are messages to be sent, i.e., the internal queue is not empty. Figure 5 shows an example of a buffer component that corresponds to port $p\left[X_{r}\right]$ (port $p$ exporting a set of variables $X_{r}$ ).

Integration. We now are ready to define the semantics of a partially asynchronous composite component $C^{\star}$ as follows: (1) create a buffer component for each receive port; (2) append ordinary interactions; (3) for each DASR interaction we create one interaction connecting the send port of the DASR interaction to the receive ports of the buffers that correspond to the receive ports of the DASR interaction, and we create one binary interaction for each receive port, which is connected to send port of its corresponding buffer. Finally, all send and receive ports in HDBIP become ordinary.

Definition 11 (PA composite component semantics). Given a partially asynchronous composite component $C^{\star}=\gamma^{\star}\left(\left\{B_{i}^{\star}\right\}_{i \in I}\right)$, its semantics is defined by the transformation into a regular BIP system $C=\llbracket C^{\star} \rrbracket$, such that $C=\gamma\left(\left\{B_{i}\right\}_{i \in I} \cup B U\right)$ where:

- $B_{i}$ is the atomic component that corresponds to $B_{i}^{\star}$ by removing labeling of the ports;

- BU is the set of buffers created for each receive port in $C^{\star}$;

- $\gamma$ is the set of interactions applied to the set of atomic components $\left\{B_{i}\right\}_{i \in I} \cup B U$ such that $\gamma=\gamma_{o} \cup \gamma_{s} \cup \gamma_{r}$ where, $\gamma_{o}$ is the set of all ordinary interactions in $C^{\star}$, $\gamma_{s}=\bigcup_{a \in \gamma_{s r}}\left\{\left(P_{a}\right.\right.$, true, identity $) \mid P_{a}=\{$ a.send $\left.\} \cup \bigcup_{r \in \text { a.recvs }}\left\{B u_{p}^{i} . r e c v\right\}\right\}$ is the set of interactions between each direct send port in interaction $a$ and the corresponding buffer receive port, and $\gamma_{r}=\bigcup_{p \in P_{r}}\left\{\left(P_{a}\right.\right.$, true, identity $) \mid p \in$ $B_{i}^{\star} . P \wedge P_{a}=\left\{p, B u_{p}^{i}\right.$.send $\left.\}\right\}$ is the set of interactions between each receive port and its corresponding send buffer port.

Figure 6 shows how the HDBIP model presented in Figure 4 is transformed to its equivalent BIP model. All the PA atomic components $B_{1}^{\star}, B_{2}^{\star}$ and $B_{3}^{\star}$ are transformed to their equivalent BIP versions (ignoring ports types) $B_{1}, B_{2}$ and $B_{3}$ respectively. For every direct receive port $\left(B_{1}^{\star} \cdot p_{0}, B_{3}^{\star} \cdot p_{0}\right)$ we added a corresponding buffer component in the BIP model. Then, the DASR interaction, in the HDBIP model, from the send port $B_{2}^{\star} \cdot p_{0}$ to $B_{1}^{\star} \cdot p_{0}$ and $B_{3}^{\star} \cdot p_{0}$ is replaced by an interaction involving $B_{2} \cdot p_{0}$ and the port receive of each of the buffer components corresponding to the receive ports of the HDBIP model. Additionally, DASR replacement includes adding other interactions involving the port send of every buffer component and its corresponding previous receive port. For this example, we included two interactions: (1) involving $B_{1} . p_{0}$ and Buffer 1.send, (2) $B_{3} \cdot p_{0}$ and Buffer2.send. 


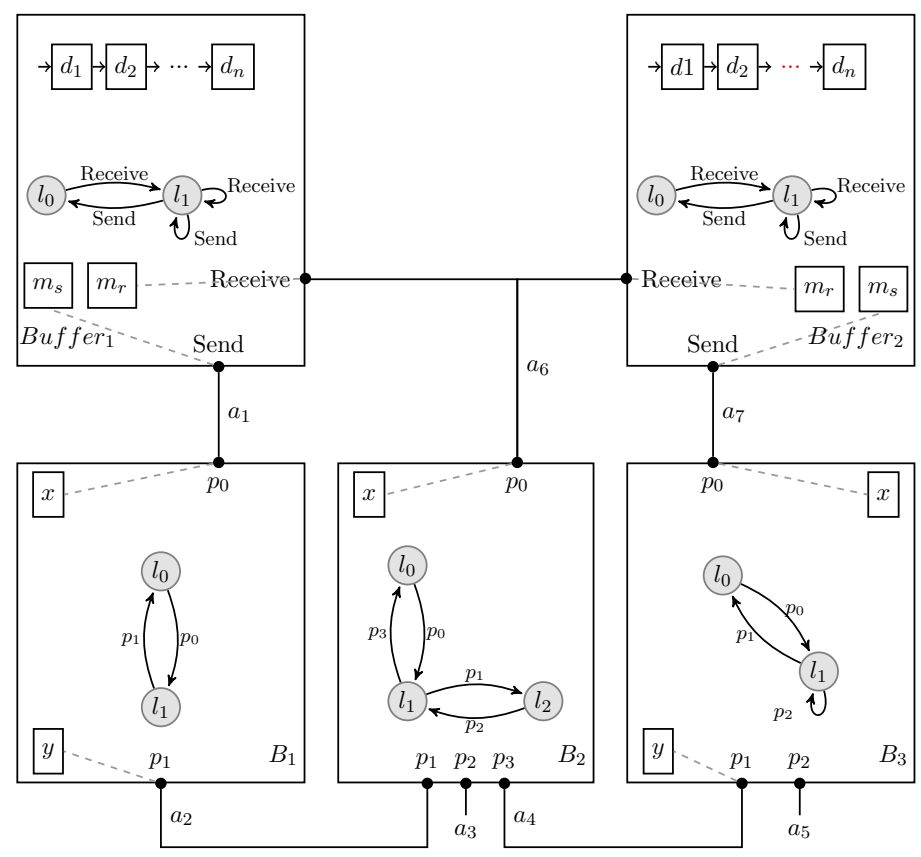

Fig. 6: Transformation of HDBIP composite in Figure 4 to BIP

\section{Efficient Code Generation}

Given an HDBIP system, it is possible to transform it to a regular BIP (i.e., consisting only of regular ports) and use the code generation provided by BIP (three-layer model). However, this may lead to the generation of inefficient implementations mainly because of: (1) the buffer components that correspond to receive ports will be replaced with actual threads or processes; (2) interactions between send/receive ports and the buffer components will be mixed with the multiparty interactions and will be added to the interaction protocol components; hence, their execution requires communication between base components, interaction protocols and possibly with conflict resolution components in case of conflicts. Although using HDBIP simplifies the development process by automatically generating buffer components and the corresponding communications, a naive implemenention would impose an additional overhead due to the extra communication as well as the creation of unnecessary threads/processes to represent the buffer components. Therefore, we introduce an efficient code generation that allows to avoid the creation of buffer components and the communication with the interaction and conflict resolution layers. To do so, we first transform PA atomic components of HDBIP system by splitting (following [6]) the transitions labeled with ordinary ports into two transitions to interact and receive notifications from the interaction protocol components, respectively. As for the transitions labeled with send and receive ports are not split and kept unchanged. Figure 7 presents an example of the transformation of a PA atomic component into its equivalent 


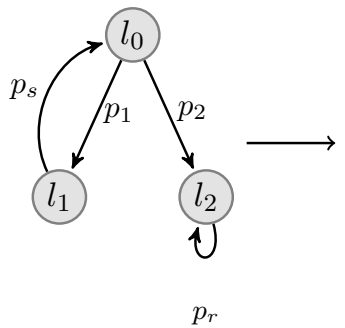

(a)

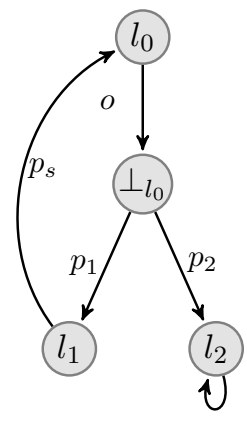

$p_{r}$

(b)

Fig. 7: HDBIP transitions transformation in 3-layer model

PA send/receive atomic component. Second, we generate the three layer send/receive model by only creating components in the interaction layer for ordinary interactions. DASR interactions are not integrated with the interaction layer and remain in the transformed model.

\subsection{Correctness}

The aim of the proof is to show that the efficient code generation is equivalent to the one provided by transforming HDBIP into regular BIP. The proof consists of two independent steps: (1) preservation of the buffer components; (2) no need for conflicts handling.

Preservation of the buffer components. Our code generation produces C++ implementation that uses MPI for the communication between threads/processes. As MPI has its internal system buffer, sending a message to a specific receive port (i.e., labeled with the name of the receive port) is implicitly added to the system buffer of MPI with the corresponding label. As such, there is no need to create buffer components.

No need for conflict handling. In the equivalent BIP model obtained from HDBIP (Definition 11), conflicts may occur between: (1) only ordinary interactions; (2) ordinary and DASR interactions; (3) only direct/send interactions. Recall that our efficient code generation only requires to integrate ordinary interactions into interaction and conflict resolution layer, whereas DASR interactions are kept unchanged in the 3-layer send/receive model. As such, conflicts between only ordinary interactions are resolved by the interaction protocol and the conflict resolution protocol layers in the usual way (Section 2). As from any state, the outgoing transitions can be labeled with either ordinary ports or send/receive ports, it is not possible to get conflicts between ordinary and direct send/receive interactions. Regarding direct send/receive 
interactions, a conflict may arise between two interactions that either involve: (1) a common direct receive port; (2) a common direct send port; (3) two ports of the same component that are the labels of two outgoing transitions from a same state. As for the (1) the execution of the receive port allows the buffer component to remain in state $l_{1}$ (see Figure 5). As such, even in the case of two concurrently-executing interactions connected to the same receive port, the final state will still belong to the state space of the semantics of the transformed regular BIP. As for (2) a direct send port can be connected to only one interaction. As for (3) we consider several cases either: (3a) the two ports are send ports, then the component will pick one of the two ports and execute the corresponding send; (3b) the two ports are receive ports, then the component can execute the corresponding receive port that has a message on its buffer, that is; (3c) one port is send and another is receive, in order to avoid deadlock of the execution, we consider giving priority to send port if its guard is enabled, otherwise, we can safely wait until a message on one of the receive ports is available. Consequently, in all the cases a conflict can be resolved locally.

\section{Performance Evaluation}

We evaluate the execution times and the number of lines of code in HDBIP versus BIP on distributed two-phase commit protocol [13]. Two-phase commit is a consensus protocol used to commit or abort a distributed transaction. A distributed transaction consists of a sequence of operations applied to several processes/participants. The system consists of $n$ resource managers (participant of the transaction) $r m_{1}, r m_{2}, \ldots, r m_{n}$ and a transaction manager $\mathrm{tm}$. Executing a distributed transaction consists of the following steps: (1) the client sends a begin transaction message to $t m$; (2) client executes the operations of the transaction on its participants (resource managers); (3) client sends a commit transaction message to $t m$; (4) $t m$ starts running two-phase commit protocol by sending a vote request message to all the resource managers; (5) each resource manager has the ability to commit or abort the transaction by sending local commit or local abort; (6) $\mathrm{tm}$ receives all the votes and broadcasts global commit to all resource managers if it has received a local commit from all the resource managers, otherwise it broadcasts global abort message; finally (7) depending on the receive message a resource manager either aborts or commits the transaction. For the sake of simplicity, we omit the handling of crash/recovery and timeouts that are handled by running specific termination protocols and by assuming the existence of persistent storage to keep track of the logs.

We provide two implementations of two-phase commit protocol using standard BIP and HDBIP. Figures $8 \mathrm{a}$ and $8 \mathrm{~b}$ show the atomic components of the clients in standard BIP and HDBIP, respectively. It mainly initiates the transaction by calling remote procedure calls on the resource managers accompanied with the current transaction id $j$. It then notifies $t m$ through the port commit and waits for the reception of the global decision. In case of standard BIP all ports are ordinary. In HDBIP only globalAbort and globalCommmit ports are ordinary as they require a global agreement (multiparty interaction), and all the other remaining ports are send ports. 


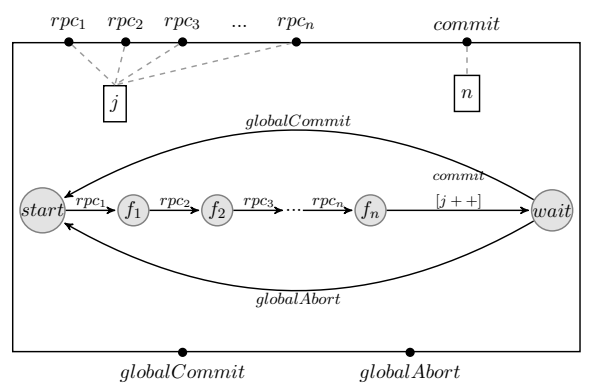

(a) Client component in BIP

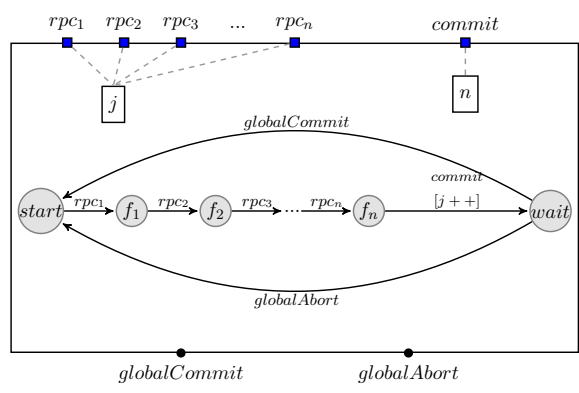

(b) Client component in HDBIP

Fig. 8: Client component in BIP and HDBIP

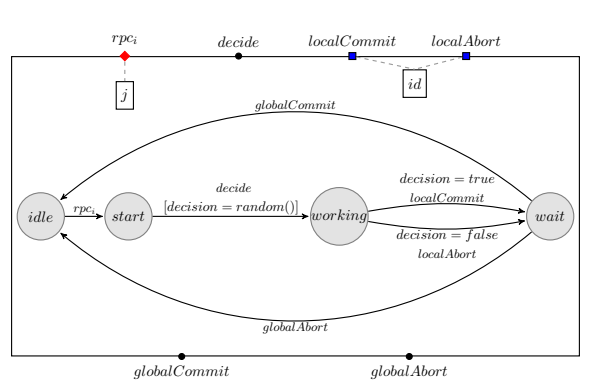

(a) Resource manager in HDBIP

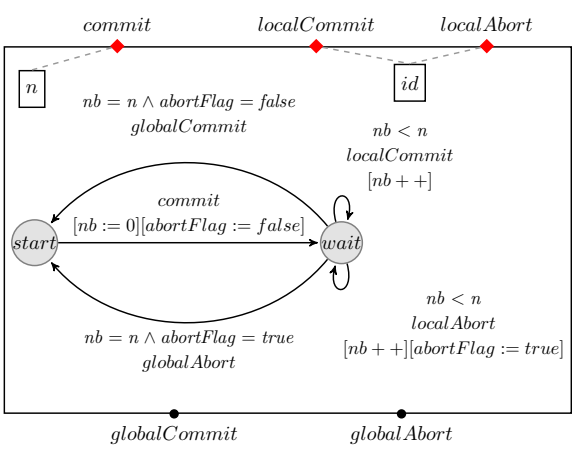

(b) Transaction manager in HDBIP

Fig. 9: Resource and transaction managers in HDBIP

The behavior of the resource manager $\mathrm{rm}$ and transaction manager $\mathrm{tm}$ in HDBIP are shown in Figures 9a and 9b (in regular BIP, we have the same behavior but all ports are ordinary). Each $\mathrm{rm}$ starts the transaction by executing the function. Then, a decision is made to abort or commit the transaction. Accordingly, it either synchronizes with $\mathrm{tm}$ with the port localCommit or localAbort. tm collects all the responses and synchronizes with all the resource managers as well as the clients to globally commit or abort the transaction.

Figures 10 and 11 show the composite component of the whole system in regular BIP and HDBIP, respectively. Recall that in regular BIP all buffers should be explicitly modeled with components and all ports are ordinary ports. In HDBIP the design is much simpler as buffer components will be implicitly replaced by the system buffers during code generation.

Efficiency. We compare the execution times of the distributed implementations generated from BIP and the one generated from HDBIP. Note that in case of HDBIP the direct send receive interactions are treated in a special way and are not integrated with the regular code generation of multiparty interactions. We consider two different 


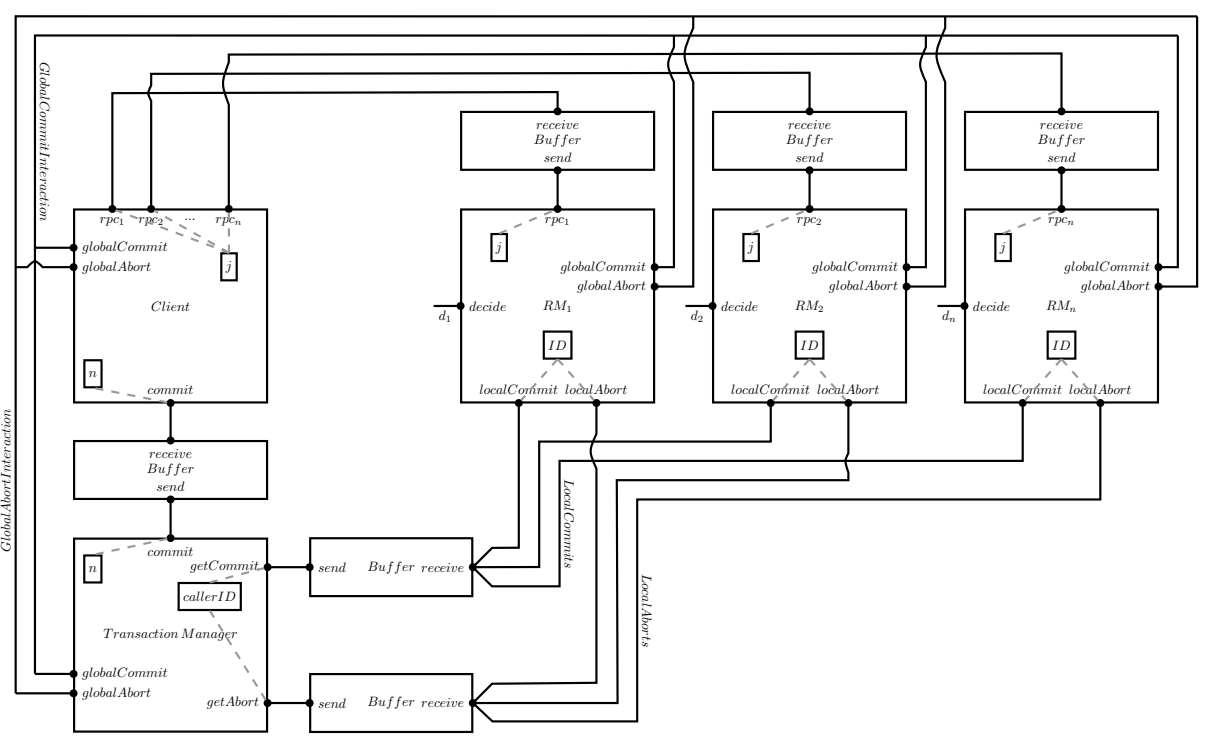

Fig. 10: Two-phase commit in BIP

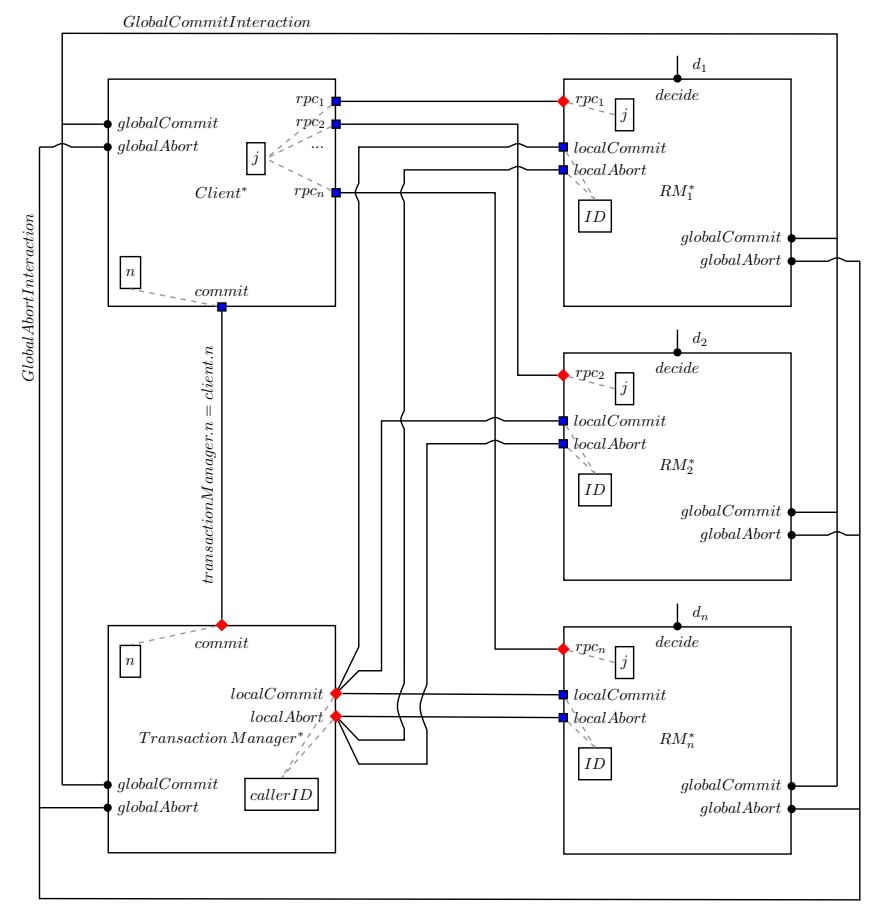

Fig. 11: Two-phase commit in HDBIP 
scenarios by varying the number of resource managers and the number of transactions. For both scenarios, we consider a cluster of four Linux machines (64-bit Ubuntu 16.04), each with 8 cores, Intel Core i7-6700 processor, and 32 GB memory. In the first scenario, we vary the number of transactions from 20,000 to 200,000 by a step of 20,000 and we fix the number of resource managers to be 10 . In the second scenario, we vary the number of resource managers from 2 to 20 by a step of 2 , and we fix the number of transactions to be 10,000 . Figures $12 \mathrm{a}$ and $12 \mathrm{~b}$ show the execution times of theses scenarios for both implementations, respectively. In both scenarios, it is clear that the implementation of HDBIP drastically outperforms regular BIP. This is mainly due to the extra messages exchanged in case of the regular BIP with the buffer components, and the multiparty interactions between the buffer components. In case of HDBIP, we can still execute multiparty interactions, however, direct send receive can be directly executed with no need to create message buffer and benefit from the system buffers that are already available.

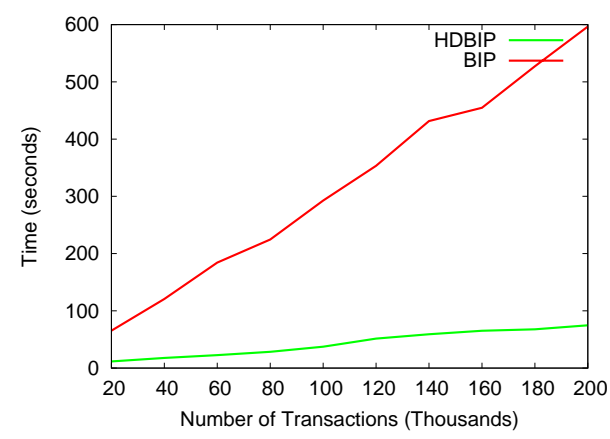

(a) Scenario 1

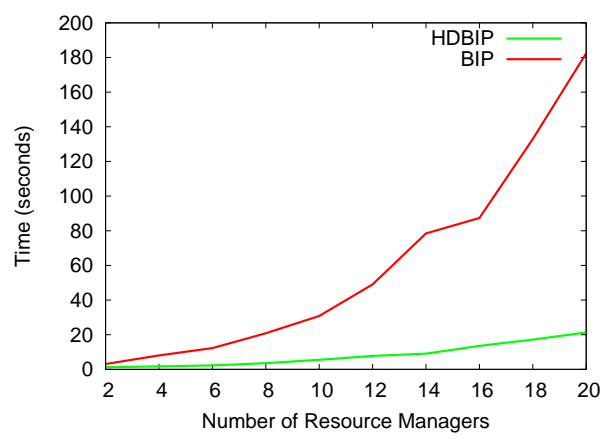

(b) Scenario 2

Fig. 12: Performance evaluation of two-phase commit

Lines of code (LOC). Using HDBIP requires less LOC than BIP as there is no need to create (1) the buffer component type and the corresponding instances; (2) the interactions between send/receive ports and the buffer components. For instance, modeling two-phase commit in case of 10 resource managers, requires 280 LOC in case of HDBIP and 390 LOC in case of BIP.

\section{Related Work}

In [11], a method is introduced to automatically generate correct asynchronously communicating processes starting from a global communication protocol. Unlike our model, the proposed method considers a simple communication model where each message has a unique sender and receiver. As such, modeling multiparty interactions requires to explicitly defining the communication protocol and conflict resolution handling, which is time consuming and error prone. 
Session types $[4,15,8,17,12]$ model interactions between distributed processes, and are based on the following methodology: (1) interactions are described as a global protocol between processes; (2) Local protocols are synthesized by projecting global protocol to local processes; (3) implementation of local processes; (4) type-checking of local types with respect to local processes. The design methodology of session type has major drawbacks: (1) there is a huge gap between design and implementation; (2) the design flow includes redundancy (global protocol, local protocol, process implementation), which is error prone; (3) there is no clear separation between communication and computation in local processes.

LASP [16] is a programming model designed to facilitate the development of reliable and large-scale distributed computing. It combines ideas from deterministic data-flow programming and conflict-free replicated data types (CRDTs). However, LASP is tailored to consistency over replicated data types. It would be interesting to integrate LASP with HDBIP to support fault-tolerance in HDBIP.

Other industrial frameworks simplify the development of large scale distributed systems such as AzureBot [1]. However, using such frameworks modeling communication models and synchronization are too abstract, which does not allow the expressiveness of explicit communication models. Moreover, AzureBot supports only applications written in $\mathrm{C} \#$ and hosted in the Azure cloud platform.

Some recent research efforts tackle correctness-preserving code generation from models to asynchronously communicating systems. For example, in AlbertBBM16,HenrioR16, they introduce a formal translation from abstract behavioral specification (ABS) to object-oriented implementation, where [2] (resp. [14]) specifically targets parallel (resp. distributed) systems. However, the underlying communication model of ABS does not support multiparty interactions but only asynchronous calls.

\section{Conclusion and Perspectives}

We introduce a rigorous model to facilitate the development of correct, efficient and scalable distributed systems. In particular, HDBIP allows both multiparty and asynchronous send/receive primitives. Moreover, our method (1) uses the primitives provided by the underlying systems such as system buffers; and (2) makes a clear separation, which is correct-by-construction, between multiparty interactions and asynchronous send/receive interactions; which allow the generation of efficient distributed implementations

For future work, we first consider to develop a source-to-source transformation from session types to HDBIP. This would avoid code redundancy of the methodology provided by session types. Moreover, we consider using other primitives provided by the underlying library (e.g., MPI) such as barriers in order to support efficient implementation of multiparty interactions. We also work on extending HDBIP to support fault tolerance. We also consider to leverage the asynchronous send/receive communication primitive to improve the efficiency of the runtime verification [10] and enforcement [9] of component-based systems. 
Acknowledgment. The authors acknowledge the support of the University Research Board (URB) at American University of Beirut and the ICT COST (European Cooperation in Science and Technology) Action IC1402 Runtime Verification beyond Monitoring (ARVI).

\section{References}

1. Agarwal, D., Prasad, S.K.: Azurebot: A framework for bag-of-tasks applications on the azure cloud platform. 2013 IEEE International Symposium on Parallel \& Distributed Processing, Workshops and Phd Forum (2013). https://doi.org/10.1109/ipdpsw.2013.261

2. Albert, E., Bezirgiannis, N., de Boer, F.S., Martin-Martin, E.: A formal, resource consumption-preserving translation of actors to haskell. In: Logic-Based Program Synthesis and Transformation - 26th International Symposium, LOPSTR 2016, Edinburgh, UK, September 6-8, 2016, Revised Selected Papers. pp. 21-37 (2016)

3. Basu, A., Bensalem, S., Bozga, M., Combaz, J., Jaber, M., Nguyen, T.H., Sifakis, J.: Rigorous component-based system design using the bip framework. IEEE Software 28(3), 41-48 (2011)

4. Bejleri, A., Yoshida, N.: Synchronous multiparty session types. Electr. Notes Theor. Comput. Sci. 241, 3-33 (2009). https://doi.org/10.1016/j.entcs.2009.06.002, http://dx. doi. org/10.1016/j.entcs.2009.06.002

5. Bliudze, S., Sifakis, J.: The algebra of connectors - structuring interaction in BIP. IEEE Trans. Computers 57(10), 1315-1330 (2008). https://doi.org/10.1109/TC.2008.26, https : //doi.org/10.1109/TC.2008.26

6. Bonakdarpour, B., Bozga, M., Jaber, M., Quilbeuf, J., Sifakis, J.: A framework for automated distributed implementation of component-based models. Distributed Computing 25(5), 383-409 (2012). https://doi.org/10.1007/s00446-012-0168-6, http://dx. doi. org/10.1007/s00446-012-0168-6

7. Bonakdarpour, B., Bozga, M., Quilbeuf, J.: Model-based implementation of distributed systems with priorities. Design Autom. for Emb. Sys. 17(2), 251-276 (2013). https://doi.org/10.1007/s10617-012-9091-0, https://doi.org/10.1007/ s10617-012-9091-0

8. Bonelli, E., Compagnoni, A.B.: Multipoint session types for a distributed calculus. In: Trustworthy Global Computing, Third Symposium, TGC 2007, Sophia-Antipolis, France, November 5-6, 2007, Revised Selected Papers. pp. 240-256 (2007)

9. Falcone, Y., Jaber, M.: Fully automated runtime enforcement of componentbased systems with formal and sound recovery. STTT 19(3), 341-365 (2017). https://doi.org/10.1007/s10009-016-0413-6, https://doi.org/10.1007/ s10009-016-0413-6

10. Falcone, Y., Jaber, M., Nguyen, T., Bozga, M., Bensalem, S.: Runtime verification of component-based systems in the BIP framework with formally-proved sound and complete instrumentation. Software and System Modeling 14(1), 173-199 (2015). https://doi.org/10.1007/s10270-013-0323-y, https://doi.org/10.1007/ s10270-013-0323-y

11. Farah, Z., Ait-Ameur, Y., Ouederni, M., Tari, K.: A correct-by-construction model for asynchronously communicating systems. Int. J. Softw. Tools Technol. Transf. 19(4), 465485 (Aug 2017)

12. Gay, S.J., Vasconcelos, V.T., Ravara, A., Gesbert, N., Caldeira, A.Z.: Modular session types for distributed object-oriented programming. In: Proceedings of the 37th ACM SIGPLANSIGACT Symposium on Principles of Programming Languages, POPL 2010, Madrid, 
Spain, January 17-23, 2010. pp. 299-312 (2010). https://doi.org/10.1145/1706299.1706335, http://doi.acm.org/10.1145/1706299.1706335

13. Gray, J., Lamport, L.: Consensus on transaction commit. ACM Trans. Database Syst. 31(1), 133-160 (Mar 2006). https://doi.org/10.1145/1132863.1132867, http://doi. $\mathrm{acm}$.org/10.1145/1132863.1132867

14. Henrio, L., Rochas, J.: From modelling to systematic deployment of distributed active objects. In: Coordination Models and Languages - 18th IFIP WG 6.1 International Conference, COORDINATION 2016, Held as Part of the 11th International Federated Conference on Distributed Computing Techniques, DisCoTec 2016, Heraklion, Crete, Greece, June 6-9, 2016, Proceedings. pp. 208-226 (2016)

15. Honda, K., Yoshida, N., Carbone, M.: Multiparty asynchronous session types. In: Proceedings of the 35th ACM SIGPLAN-SIGACT Symposium on Principles of Programming Languages, POPL 2008, San Francisco, California, USA, January 7-12, 2008. pp. 273-284 (2008). https://doi.org/10.1145/1328438.1328472, http: / / doi . acm.org/ $10.1145 / 1328438.1328472$

16. Meiklejohn, C., Van Roy, P.: Lasp: A language for distributed, coordination-free programming. In: Proceedings of the 17th International Symposium on Principles and Practice of Declarative Programming. pp. 184-195. PPDP '15, ACM, New York, NY, USA (2015). https://doi.org/10.1145/2790449.2790525, http://doi.acm.org/10.1145/ 2790449.2790525

17. Vallecillo, A., Vasconcelos, V.T., Ravara, A.: Typing the behavior of software components using session types. Fundam. Inform. 73(4), 583-598 (2006), http://iospress. metapress.com/content/82bflqafeel $5 \mathrm{~g} 8 \mathrm{n} 4 /$ 